DOI $10.35381 / \mathrm{cm} . v 7 i 1.542$

\title{
El voto electrónico alternativa para el proceso electoral ecuatoriano en tiempos de pandemia
}

\section{Alternative electronic voting for the Ecuadorian electoral process in times of pandemic}

Yovanny Monserrate Carreño-Vélez dq.yovannymcv20@uniandes.edu.ec Universidad Regional Autónoma de los Andes, Quevedo Ecuador https://orcid.org/0000-0001-9655-454X

Pamilys Milagros Moreno-Arvelo uq.pamilysmoreno@uniandes.edu.ec Universidad Regional Autónoma de los Andes, Quevedo Ecuador https://orcid.org/0000-0001-8913-4352

Rously Edyah Atencio-González uq.rouslyatencio@uniandes.edu.ec Universidad Regional Autónoma de los Andes, Quevedo Ecuador https://orcid.org/0000-0001-6845-1631

Recibido: 10 de mayo de 2021 Aprobado: 15 de agosto de 2021 


\title{
RESUMEN
}

El objetivo de la investigación fue analizar las restricciones que ha detenido la continuidad de los procesos de voto electrónico en Ecuador, a pesar de atravesar una situación de pandemia y como alternativa para el proceso electoral ecuatoriano. Se desarrolló desde la vertiente cuantitativa de tipología descriptiva, complementándose por un diseño documental-bibliográfica, por cuanto la data correspondió a los datos extraídos de diferentes textos jurídicos, trabajos arbitrados, tesis y otros. Mediante el análisis de contenido desde lo analítico-sintético, sobre el tema electoral. En definitiva, puede aceptarse al voto electrónico como una alternativa dentro de los procesos electorales en tiempo de pandemia en Ecuador. Se concluye que es necesario ampliar la legislación ecuatoriana en materia electoral vigente, lo que permitiría optimizar las ventajas del voto electrónico en situaciones de pandemia, para que brinde al ciudadano la garantía de disfrutar de su derecho al sufragio y su derecho a la salud.

Descriptores: Sistema electoral; voto; tecnología de la información; internet; derecho a la salud. (Palabras tomadas del Tesauro UNESCO).

\begin{abstract}
The objective of the investigation was to analyze the restrictions that have stopped the continuity of the electronic voting processes in Ecuador, despite going through a pandemic situation and as an alternative for the Ecuadorian electoral process. It was developed from the quantitative aspect of descriptive typology, complemented by a documentary-bibliographic design, since the data corresponded to the data extracted from different legal texts, refereed works, theses and others. Through the analysis of content from the analytical-synthetic point of view, on the electoral issue. In short, electronic voting can be accepted as an alternative within electoral processes in times of pandemic in Ecuador. It is concluded that it is necessary to expand Ecuadorian legislation on electoral matters in force, which would allow optimizing the advantages of electronic voting in pandemic situations, so that it provides citizens with the guarantee of enjoying their right to vote and their right to health.
\end{abstract}

Descriptors: Electoral system; vote; information technology; Internet; right to health. (Words taken from the UNESCO Thesaurus). 


\section{INTRODUCCIÓN}

Es indudable que en la actualidad las Tecnologías de la Información y la Comunicación(TIC) se han convertido en aliadas ante una realidad global de confinamiento y pandemia producto del SARS-CoV-2 y la misma desempeña un rol protagónico en el desarrollo de los procesos electorales, estudios han demostrado las crecientes ventajas que estas tecnologías tienen en este tipo de procesos, numerosos países han optado por tomarlo como una opción, sin embargo existe un debate de criterios, por considerar que más que ventajas llevar las TIC a los procesos de sufragio, podrían desencadenar en situaciones de fraudes masivos y en red. Al respecto Bizberge y Segura (2020) mencionan:

Las nuevas necesidades y problemas que se presentan durante el avance de la primera pandemia global de coronavirus (COVID-19) y las excepcionales medidas de aislamiento de la población adoptadas en un tercio de los países del mundo hicieron resurgir los debates públicos sobre los derechos digitales. Además, pasaron del estrecho círculo de especialistas a ser parte de la discusión social, mediática y política. (p.62)

A esta realidad no escapa el Estado ecuatoriano, quien en este contexto tuvo su primera experiencia de voto electrónico en el año 2013, en las Parroquias La Esmeralda y Panguintza, para escoger a las autoridades parroquiales, para ese momento una empresa española fue la encargada de proveer al país de todo el sistema digital de datos utilizado para los comicios, sin embargo, no se cuenta con mucha información por parte del Consejo Nacional Electoral al respecto. (Castillo, 2015 citado por Torres Bonilla, 2017).

Si bien es cierto la experiencia en el país del año 2013, fue positiva no obstante este sistema de voto electrónico genera una inversión del Estado ecuatoriano elevada en comparación con el voto manual, lo que podría considerarse una de las desventajas de estos procesos, esto podría entenderse como una contradicción si se trata de verificar todas las ventajas que brinda este mecanismo, donde a la luz de esferas internacionales, el voto electrónico ha evolucionado y en el país ecuatoriano, sí se implementan las Tecnologías de la Información y la Comunicación (TIC) en diferentes momentos del proceso. Al respecto González de la Garza (2009) manifiesta: 
La introducción de la informática en el proceso electoral —más allá de la tabulación de los grandes resultados agregados- es un problema crítico en cualesquiera de las dimensiones operativas del voto electrónico, que genera un reto de excepcional magnitud tanto para el «proceso electoral» como para el régimen electoral en su conjunto. (164)

Sin embargo, la gran mayoría de los países está optando por dirigir sus procesos electorales haciendo uso de las ventajas que ofrecen las Tecnologías de la Información y la Comunicación (TIC), en este sentido, Torres Bonilla (2017) plantea lo siguiente:

El voto electrónico se viene implementado en diversos lugares del mundo, con experiencias diversas, pero el mayor obstáculo a considerar se relaciona con el desempeño por parte de los organismos electorales, se debe tener en cuenta que el éxito de la adopción de un sistema de votación electrónica depende de muchísimos factores, pero sobre todo la transparencia y la confiabilidad con el fin de ofrecer resultados incuestionables. (p.16)

Por otro lado, está la votación a través del internet, en la cual se hace uso de una computadora personal con conexión a internet para emitir el voto, el cual es dirigido a una computadora remota, la cual lo almacena, por otra parte, el voto electrónico también puede apoyarse en agendas electrónicas y en teléfonos fijos o móviles para ser procesado, lo que indica que se apoya en este tipo de herramientas tecnológicas. Así mismo, es imperioso que los estados y especialmente Ecuador permita que sus ciudadanos ejerzan el derecho al voto a través de medios electrónicos, sin tomar en consideración de qué manera se haga uso de la tecnología, o de qué sistema se utilice o si se usan esas tecnologías en cualquier fase del proceso electoral, que no implique la votación como tal y en este sentido se considera al voto electrónico como una gran alternativa viable y factible para que los ciudadanos ejerzan su derecho al voto de manera remota.

En Ecuador, esta iniciativa del voto electrónico a raíz de la pandemia que atraviesa la humanidad completa, se ha extendido inclusive a sectores de la Academia, siendo así la Universidad Técnica del Norte, planteó sus elecciones de Cogobierno 2021 
mediante votación electrónica, para ello se apoyaron en los correos institucionales de su personal docente y administrativo, instalando las Juntas receptoras de votos a través de la plataforma Teams, evidenciándose entonces que estos procesos garantizan ese derecho al voto que tienen las personas y para lo cual se utilizan las TIC en todos sus procesos.

En contrates a lo planteado, según Hernández Trejo (2019) el buen uso de la tecnología:

debe ser dimensionada como un instrumento, una herramienta de comunicación e información que puede seguir siendo percibida como un incentivo dentro de la política para la participación ciudadana, la deliberación y la toma de decisión ciudadana, pero no es por sí misma el "botón de cambio"; no es, pues, inherentemente democrática. (p.63)

Las ideas anteriormente planteadas, quizás no se refiera a lo que es el sufragio y el derecho al voto de los ciudadanos, orientado en cada uno de los procesos de elecciones por el Consejo Nacional Electoral (CNE), sin embargo, demuestra las alternativas que en Ecuador se han optado en una situación de pandemia global para canalizar procesos de elecciones en distintos contextos. Cabe señalar, que, si esta es una realidad, el voto electrónico ofrece grandes ventajas, por ejemplo, podría contribuir a disminuir las probabilidades de fraudes electorales, tener un acceso mucho más eficaz y oportuno a los resultados, reducir al Estado los costos de los procesos y referendos, pero sin olvidar que las tecnologías también pueden presentar problemas, donde en algunos casos expertos en la materia consideran que los sistemas operativos pueden ser manipulados.

Es importante mencionar que, el voto electrónico contribuye entre otras cosas a reducir los márgenes de error, hacen los procesos más rápidos, evita que se den los famosos votos nulos que sesgan los resultados, además de ser una gran alternativa para ciudadanos que cuenten con alguna discapacidad o que se encuentren fuera del país, por cualquier motivo en el momento de los comicios. La democracia electrónica ha involucrado, por un lado, una serie de reformas que utilizando los nuevos medios 
tecnológicos disponibles han intentado entregar soluciones novedosas a problemas democráticos actuales. (Marshall Barberan, 2018, p.264)

En el contexto de la Pandemia del Covid-19, el Consejo Nacional para la Igualdad de Género, (2020) remitió un oficio donde se solicitaba analizar las distintas modalidades y escenarios en que se podrían llevar a cabo las elecciones del 2021, ante una crisis sanitaria, incluso planteando una síntesis de los procesos electorales telemáticos realizados en otros países, planteando los pros y los contras e inclusive los costos políticos que generaron esos procesos. Se planteó el caso de República Dominicana, donde se suspendieron las elecciones por fallos en el voto automatizado, lo que generó pérdidas económicas y crisis institucional, a este país latinoamericano.

En el documento que se viene planteando, se consideran varias alternativas a considerar en el contexto de emergencia sanitaria: "la votación electrónica, la votación telemática, la votación segmentada, votación mixta o híbrida que es aquella que combina diferentes formas de votación", esto a criterio del (Consejo Nacional Electoral para la Igualdad de Género, 2020)

De acuerdo a los planteamientos realizados el objetivo de la investigación es analizar las restricciones que han detenido la continuidad de los procesos de voto electrónico en Ecuador, a pesar de atravesar una situación de pandemia.

\section{METODOLOGÍA}

El presente estudio se desarrolló desde la vertiente cuantitativa de tipología descriptiva, complementándose por un diseño documental-bibliográfico, debido a la recolección de datos extraídos de diversos textos, (Parela Stracuzzi y Martins Pestana, 2012) permitió la recogida de información extraída de las diferentes citas jurídicas y trabajos científicos, emprendiendo el método analítico enfocándose en el estudio de aspectos particulares, examinados en el análisis de contenido escrito, mediante lo analítico-sintético (Bernal-Torres, 2006), adquiriendo nuevos conocimientos de la descomposición mental en varios elementos, sobre el tema electoral. 


\section{RESULTADOS}

Según informe del Consejo Nacional Electoral para la Igualdad de Género (2020) en los trámites de preparación para las elecciones de 2021 de Ecuador, se plantearon, varias alternativas que se irán detallando a continuación y que permitirán evidenciar, cómo se observa al voto electrónico como alternativa en tiempos de pandemia.

En este orden de ideas se señala al voto electrónico, el cual permite realizar el proceso de elección a través de un computador, en un lugar determinado y adaptado para estos efectos, lo que requiere la presencia física del elector. Sin embargo, se consideró que esta modalidad, es poco atractiva por el riesgo de contagio ya que implica necesariamente asistir a los lugares asignados para la votación. El mismo, cuenta con más desventajas que ventajas, en virtud de que no disminuye los riesgos de contagio, requiere inversión en la implementación de medidas de bioseguridad al personal y los ciudadanos, desinfección de espacios y respaldo de diferentes instituciones para mantener el orden público y evitar aglomeraciones. Por otro lado, como ventaja presenta que, en relación a los costos, estos son menores.

Otra alternativa planteada, es el voto telemático, esta votación se realiza a través de la internet y como su gran ventaja no requiere la presencia física del elector, sin embargo, entre sus desventajas se encuentra el hecho de que no utiliza los datos del Instituto Nacional de Estadísticas y Censos (INEC), lo que genera gran desconfianza en sus resultados.

Continuando con lo que se viene planteando, una sugerencia adicional es el voto segmentado, bosquejando que las elecciones se realicen en diferentes días, en los cuales se implementarían estrategias para prevenir el contagio de los ciudadanos, sin embargo entre sus principales debilidades se encuentra el hecho de que no se garantiza la conducta de los electores, sin tener apoyo de instituciones del Estado que velen por el cumplimiento de las medidas de bioseguridad, lo que supone poner en riesgo inminente a la población.

Siguiendo con los planteamientos se tiene la votación híbrida, la principal desventaja que presenta son los costos, sin embargo, este mecanismo requiere de espacios presenciales y no presenciales para la votación lo que indudablemente no garantiza 
la seguridad ciudadana y evitar contagios masivos del virus, por lo tanto, en algunos casos se considera como un mecanismo exitoso y en otros se le señalan cuestionamientos.

Ahora bien, ¿qué circunstancias se presentan en la realidad ecuatoriana en relación al voto electrónico como alternativa en tiempos de pandemia?, si bien es cierto desde marzo 2020, se decreta un estado de excepción, a raíz de la grave crisis sanitaria en el país, obligando a la población a confinarse, acudiendo al teletrabajo y la teleeducación, como opción para no detener al país.

Cabe señalar que, es cierto que los medios electrónicos y digitales cuentan con gran aceptación y acogida, sin embargo es necesario identificar las brechas digitales del Estado ecuatoriano, lo que genera vulneración de derechos en muchos casos, inclusive se vulnera el derecho al voto de los ciudadanos, lo que hace que se requiera el apoyo de organismos del propio Estado, para lograr difundir la información que rodea los procesos electorales, con la finalidad de que la población esté al tanto, de las ventajas y desventajas de cada una de las alternativas que se han planteado.

Ahora bien, en el siguiente cuadro observaremos algunos artículos relacionados al tema electoral en el cual se basa el sistema ecuatoriano.

\section{Cuadro 1.}

Norma electorale.

\begin{tabular}{|c|c|c|}
\hline Ley vigente & Articulado & Discusión \\
\hline $\begin{array}{l}\text { Ley Orgánica Electoral, } \\
\text { Código de la Democracia } \\
\text { (2009) }\end{array}$ & $\begin{array}{l}\text { Artículo. 18.- La Función } \\
\text { Electoral garantiza el } \\
\text { ejercicio de los derechos } \\
\text { políticos que se expresan a } \\
\text { través del sufragio, así como } \\
\text { los referentes a la } \\
\text { organización política de la } \\
\text { ciudadanía. La Función } \\
\text { Electoral estará conformada } \\
\text { por el Consejo Nacional } \\
\text { Electoral y el Tribunal } \\
\text { Contencioso Electoral. } \\
\text { Estos órganos tendrán sede } \\
\text { en Quito, jurisdicción }\end{array}$ & $\begin{array}{l}\text { La responsabilidad de } \\
\text { plantear y organizar baso } \\
\text { estrictas normas de } \\
\text { bioseguridad la participación } \\
\text { ciudadana recae en el } \\
\text { órgano electoral y } \\
\text { respetando los principios de } \\
\text { autonomía, independencia, } \\
\text { publicidad, transparencia, } \\
\text { equidad, interculturalidad, } \\
\text { paridad de género, } \\
\text { celeridad, probidad, certeza, } \\
\text { eficacia, eficiencia, calidad, } \\
\text { coordinación, planificación, }\end{array}$ \\
\hline
\end{tabular}




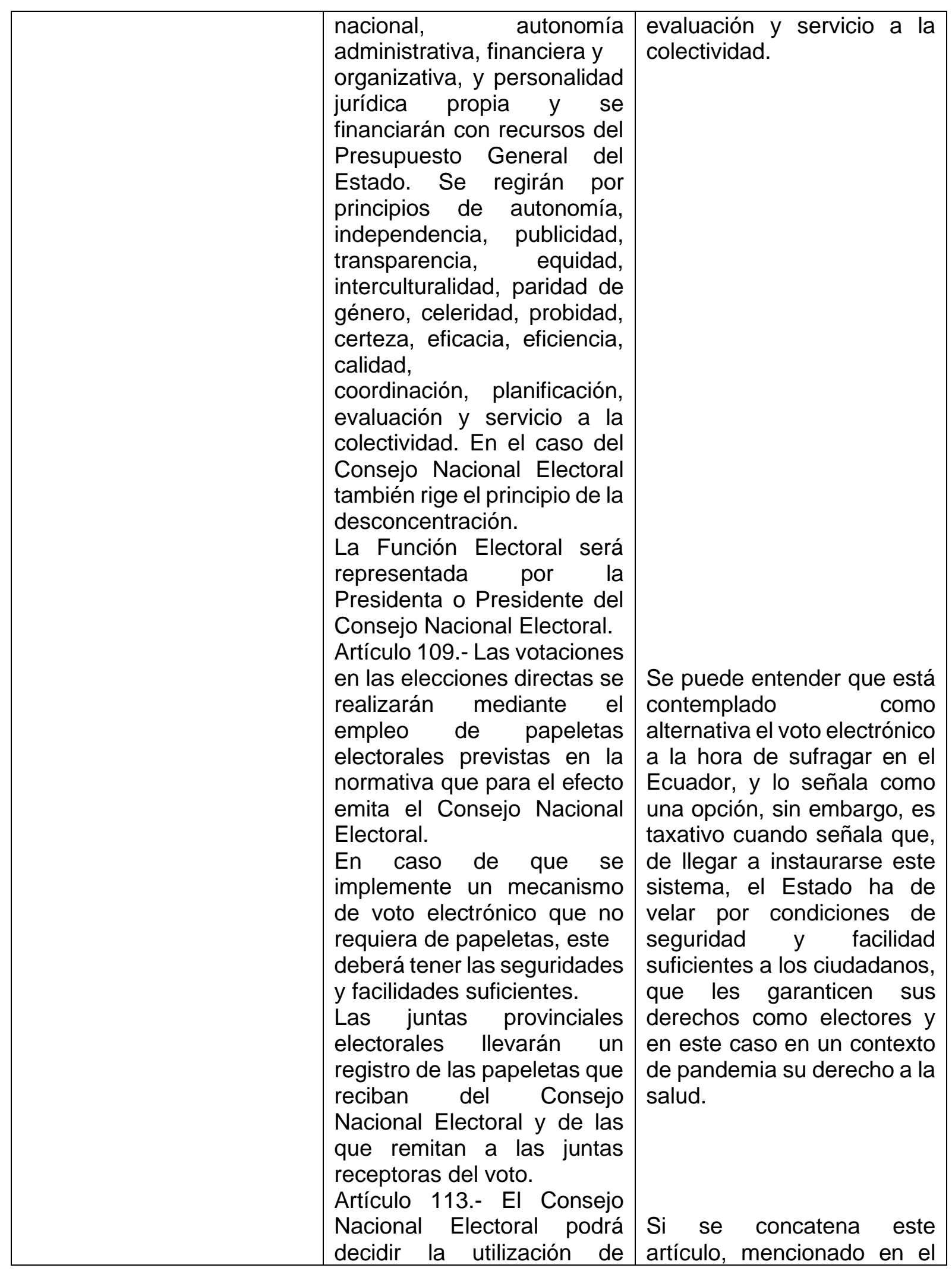




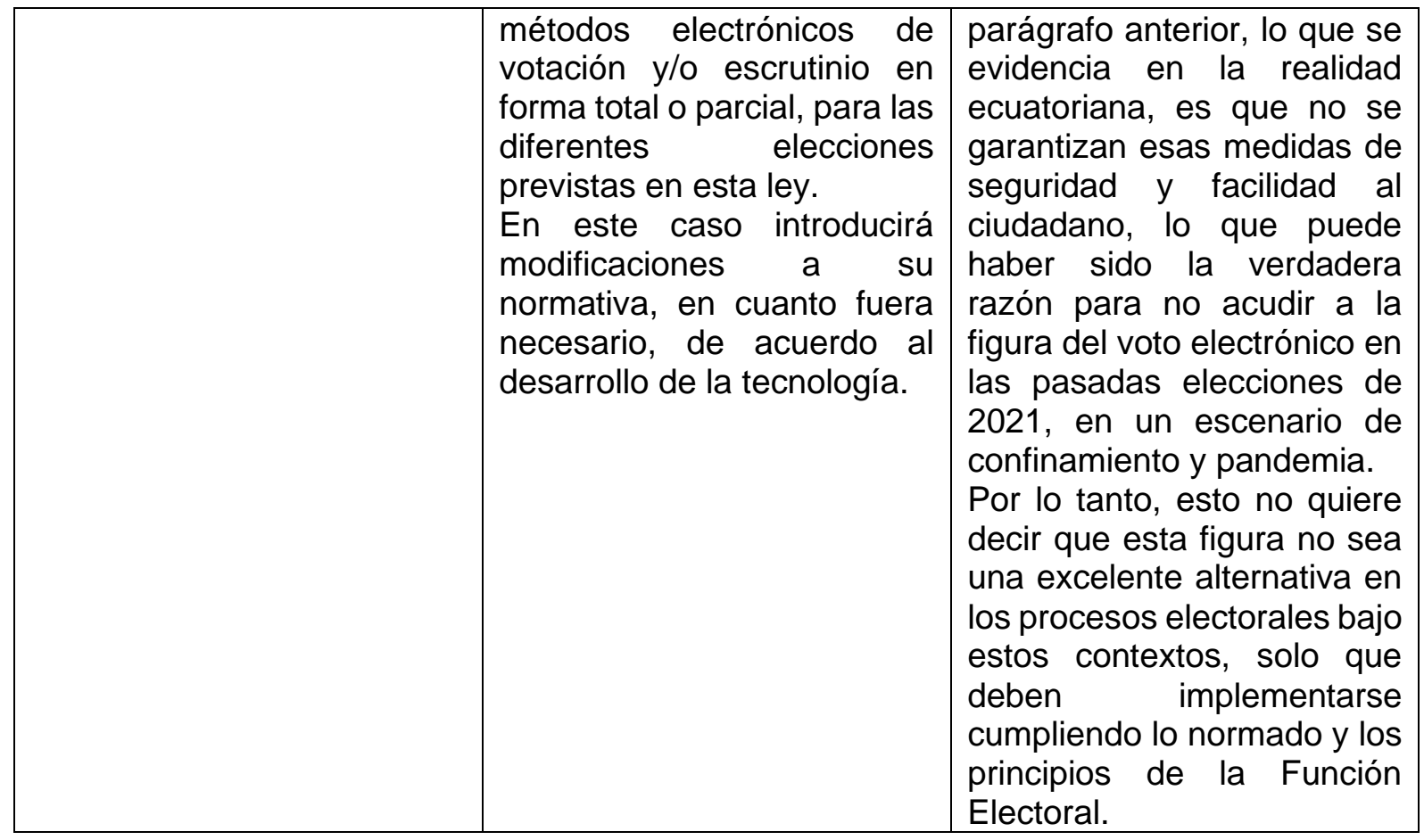

Elaboración: Los autores.

\section{DISCUSIÓN}

Implementar el voto electrónico, es un proceso que requiere de planificación, coordinación, e inversión por parte del Estado ecuatoriano, aunado a esto por la situación de emergencia sanitaria, inversión en mecanismos de bioseguridad para los ciudadanos y personal que intervienen en el proceso de sufragar y en la logística de los comicios, es por ello que se debe impulsar todo un proceso desde lo normativo hasta lo institucional para que se puedan disfrutar de las ventajas que este sistema ofrece.

Los procesos electorales, son acontecimientos sociales que mueven gran cantidad de personas y se entienden como operaciones costosas y gravosas desde lo administrativo y logístico, la pandemia vino a poner a prueba todos los elementos que están involucrados en estos procesos, donde la presencia física de los electores representa riesgos para todas las personas que sufragan y para todo el personal 
electoral, es por ello que se han de considerar ciertos requisitos por parte de los encargados de la toma de decisiones en el Ecuador.

En definitiva, puede aceptarse al voto electrónico como una alternativa dentro de los procesos electorales en tiempo de pandemia en Ecuador, lo importante es reflexionar en cuanto a que se ha de incluir en la normativa, específicamente en la Ley Orgánica Electoral, Código de la Democracia(2009), no solo la figura de la votación electrónica como está contemplado en la Sección Segunda, específicamente en el artículo 113, sino ampliar esta sección, en el sentido de especificar, bajo qué condiciones se debe instaurar cuando se trate de una situación de pandemia y comprometer a los organismos del Estado necesarios a participar activamente en la viabilidad de este proceso.

\section{CONCLUSIONES}

El voto electrónico es aquel que utiliza medios electrónicos para sufragar y en donde en el acto en sí, se requiere de un instrumento tecnológico y el involucramiento de las Tecnologías de la Información y la Comunicación (TIC). El mismo presenta grandes ventajas como la celeridad a la hora de informar los resultados, reducción de emisión de votos nulos, e involucra a personas con alguna discapacidad o que se encuentren fuera del país, por lo tanto, es más inclusivo.

La realidad del Estado ecuatoriano, en relación a tomar el voto electrónico como alternativa en sus procesos electorales en tiempos de pandemia, evidencia que se deben impulsar mecanismos, que favorezcan la viabilidad de este sistema y que contribuyan al logro de los principios de la Función Electoral y garantice la seguridad tanto del derecho al voto como la salud de los ciudadanos, además de que la legislación, en materia electoral vigente, presenta grandes vacíos en relación a la votación electrónica en situaciones de pandemia y crisis sanitaria.

Desde el análisis crítico reflexivo, se concluye que es necesario ampliar la legislación ecuatoriana en materia electoral vigente, lo que permitirá optimizar las ventajas del voto electrónico en situaciones de pandemia, para ser visto como una alternativa que brinde al ciudadano la garantía de disfrutar de su derecho al sufragio y su derecho a 
la salud, por lo tanto se recomienda una revisión a la Ley Orgánica Electoral, Código de la Democracia (2009) y que la misma ofrezca ante la figura de la votación electrónica mejores alternativas al Ecuador.

\section{FINANCIAMIENTO}

No monetario.

\section{AGRADECIMIENTO}

A la Universidad Regional Autónoma de Los Andes, sede Quevedo; por motivar el desarrollo de la Investigación.

\section{REFERENCIAS CONSULTADAS}

Asamblea Nacional (2009) Ley Orgánica Electoral, Código de la Democracia. Ley 2 Registro Oficial Suplemento 578 de 27-abr.-2009.Recuperado de: https://n9.cl/rz5y

Bernal-Torres, C. (2006). Metodología de la investigación. Recuperado de: https://n9.cl/8txy.

Bizberge, A., y Segura, M. (2020). Los derechos digitales durante la pandemia COVID19 en Argentina, Brasil y México. Revista de Comunicación, 19(2), 6185. https://dx.doi.org/10.26441/rc19.2-2020-a4

Consejo Nacional Electoral (2013) La experiencia del voto electrónico. Recuperado de: https://n9.cl/vwsqx

Consejo Nacional para la Igualdad de Género, (2020). Recomendaciones Informes del Consejo Nacional Electoral respecto a Elecciones 2021. Quito-Ecuador. Recuperado de: https://n9.cl/fja6y

González de la Garza, L. M. (2009). Voto electrónico por Internet y riesgos para la democracia. Revista De Derecho Político, (74), 163-209. https://doi.org/10.5944/rdp.74.2009.9070

Hernández Trejo, N. (2019). El voto electrónico en la construcción de un modelo de democracia electrónica. Estudios politicos, (47), 61-85. https://doi.org/10.22201/fcpys.24484903e.2019.47.69500 
Marshall Barberan, Pablo. (2018). Sufragio por Internet: análisis teórico y comparado. Cuestiones constitucionales, (39), 261-293. https://doi.org/10.22201/iij.24484881e.2018.39.12656

Palella Stracuzzi, S. y Martins Pestana, F. (2012). Metodología de la investigación cuantitativa. Fondo editorial de la Universidad Pedagógica Libertador. Caracas, Venezuela.

Torres Bonilla, C. (2017) El Sistema tradicional electoral vulnera los derechos de participación establecidos en la Ley Orgánica Electoral, en el Distrito Metropolitano de Quito, año 2014. [ The traditional electoral system violates the rights of participation established in the Organic Electoral Law, in the Metropolitan District of Quito, year 2014]. Proyecto de investigación previo a la obtención del Título de: Abogado. Universidad Central Del Ecuador. Recuperado de: https://n9.cl/u2z4 